\title{
Some optical soliton solutions for the generalized Fokas-Lenells equation
}

\author{
Esma Ulutas
}

Department of Electronics and Communication Engineering, Technology of Faculty, Trabzon, Turkey

Received: 13 June 2021, Accepted: 16 June 2021

Published online: 12 July 2021.

\begin{abstract}
In this article, we have considered the Fokas-Lenells equation with full nonlinearity. By the help of three types Jacobi elliptic functions, we have obtained bright, dark and singular optical soliton solutions of the Fokas-Lenells equation. We have also presented the constraint conditions which guarantee the existence of obtained solutions.
\end{abstract}

Keywords: Fokas Lenells equation, optical solitons, Jacobi elliptic functions.

\section{Introduction}

Soliton propagation studies in optical fibers have been flourishing because of the rich features of models describing these phenomena over the past few decades. In the field of optics, by careful selection of higher order nonlinear and linear effects, Fokas-Lenells equation(FLE) emerges as a model governing femtosecond pulse propagation through single mode silica optical fiber. Accordingly it is a useful model to understand the physical insight of ultra short pulses in media. It is one of the models that govern pulse transmissions technology first appeared about a decade ago. The existence of solitons in the nonlinear dispersive media has been studied within the framework of the FLE. Recently some research work have been conducted on exact solutions of this equation $[1,2,3,4,5,6,7,8,9,10,11]$. Here, the model is considered in the presence of perturbation terms that provide a picture much closer to reality. The FLE is studied with full nonlinearity in the generalized form using Jacobi elliptic functions in this work. It is obtained Jacobi elliptic function solutions and bright, dark and singular optical soliton solutions are also attained with the help of modulus limit of Jacobi elliptic functions for FLE. These solitons appear with constraint conditions on their parameters and they are also presented

\section{Mathematical analysis}

In the presence of dual dispersion, the dimensionless form of FLE with perturbation is given as

$$
i q_{t}+a_{1} q_{x x}+a_{2} q_{x t}+|q|^{2}\left(b q+i \sigma q_{x}\right)=i\left[\alpha q_{x}+\lambda\left(|q|^{2 m} q\right)_{x}+\mu\left(|q|^{2 m}\right)_{x} q\right] .
$$

In (1), $q(x, t)$ describes the profile of optical pulse and the first term is the temporal evolution of the pulses.The coefficients $a, b$ are real valued constants describing group velocity dispersion (GVD) and spatio-temporal dispersion (STD) respectively.

$$
q(x, t)=u(\xi) e^{i \phi}, \quad \xi=x-v t
$$

\footnotetext{
* Corresponding author e-mail: esmaates@ktu.edu.tr
} 
where $u(\xi)$ and $v$ represent amplitude and velocity of the soliton respectively while the phase component is defined as

$$
\phi=-\kappa x+\omega t+\theta
$$

where $\kappa$ is the soliton frequency, $\omega$ is the wave number and $\theta$ is the soliton phase constant. Substituting (2) into (1) and separating it into two parts real and imaginary parts yields

$$
\begin{gathered}
\left(a_{1}-a_{2} v\right) u^{\prime \prime}-\left(\omega+a_{1} \kappa^{2}-\omega a_{2} \kappa+\alpha \kappa\right) u+(\kappa \sigma+b) u^{3}-\kappa \lambda u^{2 m+1}=0 \\
\left(v a_{2} \kappa+\omega a_{2}-v-2 a_{1} \kappa-\alpha\right) u^{\prime}+\sigma u^{\prime} u^{2}+[\lambda(2 m+1)+2 m \mu] u^{\prime} u^{2 m}=0 .
\end{gathered}
$$

respectively. From the imaginary part we obtain

$$
\begin{gathered}
m=1, \\
\sigma=(2 m+1) \lambda+2 m \mu, \\
v=\frac{\omega a_{2}-2 a_{1} \kappa-\alpha}{1-a_{2} \kappa}
\end{gathered}
$$

So, real part reduces

$$
\left(a_{1}-a_{2} v\right) u^{\prime \prime}-\left(\omega+a_{1} \kappa^{2}-\omega a_{2} \kappa+\alpha \kappa\right) u+(\kappa \sigma+b-\kappa \lambda) u^{3}=0,
$$

The initial hypothesis is

$$
u(\xi)=A s n^{p}(B \xi, \ell)
$$

where $s n$ is the Jacobi elliptic function, $\ell$ is the modulus of Jacobi elliptic function and $0<\ell<1$. $A$ and $B$ represent amplitude and inverse width of the soliton. Eq. (9) reduces

$$
\begin{gathered}
\left(a_{1}-a_{2} v\right)(\rho-1) \rho A B^{2} s n^{\rho-2}(B \xi, \ell) \\
-\left(a_{1}-a_{2} v\right) \rho\left[\ell^{2}(\rho-1)+\ell+\rho\right] A B^{2} s n^{\rho}(B \xi, \ell) \\
+\left(a_{1}-a_{2} v\right) \ell \rho(\ell \rho+1) A B^{2} s n^{\rho+2}(B \xi, \ell) \\
-\left(\omega+a_{1} \kappa^{2}-\omega a_{2} \kappa+\alpha \kappa\right) A s n^{\rho}(B \xi, \ell) \\
+(\kappa \sigma+b-\kappa \lambda) A^{3} s n^{3 \rho}(B \xi, \ell)=0 .
\end{gathered}
$$

From Eq. (11), matching the exponents $s n^{\rho+2}(B \xi, \ell)$ and $s n^{3 p}(B \xi, \ell)$ yields

$$
p+2=3 p
$$

which gives

$$
p=1
$$


Equating coefficients of them and setting coefficients of $s n^{\rho+j}(B \xi, \ell)$, for $j=-2,0$, to zero in (11) as these are linearly independent functions we obtain

$$
\begin{gathered}
A=\sqrt{\frac{\ell\left(\omega+a_{1} \kappa^{2}-\omega a_{2} \kappa+\alpha \kappa\right)}{(\kappa \sigma+b-\kappa \lambda)}}, \\
B=\sqrt{\frac{\omega+a_{1} \kappa^{2}-\omega a_{2} \kappa+\alpha \kappa}{\left(a_{2} v-a_{1}\right)(\ell+1)}},
\end{gathered}
$$

So, the Jacobi elliptic function solution is obtained as follows:

$$
\begin{gathered}
q(x, t)=\sqrt{\frac{\ell\left(\omega+a_{1} \kappa^{2}-\omega a_{2} \kappa+\alpha \kappa\right)}{(\kappa \sigma+b-\kappa \lambda)}} s n\left[\sqrt{\frac{\omega+a_{1} \kappa^{2}-\omega a_{2} \kappa+\alpha \kappa}{\left(a_{2} v-a_{1}\right)(\ell+1)}}\right. \\
\left.\left(x-\frac{\omega a_{2}-2 a_{1} \kappa-\alpha}{1-a_{2} \kappa} t\right), \ell\right] . e^{i(-\kappa x+\omega t+\theta)},
\end{gathered}
$$

which requires the constraints

$$
\begin{gathered}
\left(\omega+a_{1} \kappa^{2}-\omega a_{2} \kappa+\alpha \kappa\right)(\kappa \sigma+b-\kappa \lambda)>0, \\
\left(\omega+a_{1} \kappa^{2}-\omega a_{2} \kappa+\alpha \kappa\right)\left(a_{2} v-a_{1}\right)>0 .
\end{gathered}
$$

When the modulus $\ell \rightarrow 1$ in Eq. (16), dark optical soliton solution is obtained as:

$$
\begin{gathered}
q(x, t)=\sqrt{\frac{\omega+a_{1} \kappa^{2}-\omega a_{2} \kappa+\alpha \kappa}{\kappa \sigma+b-\kappa \lambda}} \tanh \left[\sqrt{\frac{\omega+a_{1} \kappa^{2}-\omega a_{2} \kappa+\alpha \kappa}{2\left(a_{2} v-a_{1}\right)}}\right. \\
\left.\left(x-\frac{\omega a_{2}-2 a_{1} \kappa-\alpha}{1-a_{2} \kappa} t\right)\right] \cdot e^{i(-\kappa x+\omega t+\theta)} .
\end{gathered}
$$

In order to construct exact solutions for Eq. (1), we use hypothesis in the form

$$
u(\xi)=A c n^{\rho}(B \xi, \ell)
$$

From (20), Eq. (9) reduces to

$$
\begin{aligned}
& \left(a_{1}-a_{2} v\right)\left(1-\ell^{2}\right)(\rho-1) \rho A B^{2} c n^{\rho-2}(B \xi, \ell)+\left(a_{1}-a_{2} v\right) \rho\left[\ell^{2}(2 \rho-1)+\ell-\rho\right] A B^{2} c n^{\rho}(B \xi, \ell) \\
& -\left(a_{1}-a_{2} v\right) \ell \rho(\ell \rho+1) A B^{2} c n^{\rho+2}(B \xi, \ell)-\left(\omega+a_{1} \kappa^{2}-\omega a_{2} \kappa+\alpha \kappa\right) A c n^{\rho}(B \xi, \ell) \\
& +(\kappa \sigma+b-\kappa \lambda) A^{3} c n^{3 \rho}(B \xi, \ell)=0 .
\end{aligned}
$$

Setting the exponents and coefficients of functions $c n^{\rho+2}(B \xi, \ell)$ and $c n^{3 \rho}(B \xi, \ell)$ equal to one another, and then setting the coefficients functions of $c n^{\rho+j}(B \xi, \ell)$ to zero for $j=-2,0$, we acquire the same value of which is in (13) and following equations

$$
\begin{gathered}
A=\sqrt{\frac{\ell(\ell+1)\left(\omega+a_{1} \kappa^{2}-\omega a_{2} \kappa+\alpha \kappa\right)}{\left(\ell^{2}+\ell-1\right)(\kappa \sigma+b-\kappa \lambda)}} \\
B=\sqrt{\frac{\omega+a_{1} \kappa^{2}-\omega a_{2} \kappa+\alpha \kappa}{\left(a_{1}-a_{2} v\right)\left(\ell^{2}+\ell-1\right)}}
\end{gathered}
$$


So, the Jacobi elliptic function solution is obtained as

$$
\begin{gathered}
q(x, t)=\sqrt{\frac{\ell(\ell+1)\left(\omega+a_{1} \kappa^{2}-\omega a_{2} \kappa+\alpha \kappa\right)}{\left(\ell^{2}+\ell-1\right)(\kappa \sigma+b-\kappa \lambda)}} c n\left[\sqrt{\frac{\omega+a_{1} \kappa^{2}-\omega a_{2} \kappa+\alpha \kappa}{\left(a_{1}-a_{2} v\right)\left(\ell^{2}+\ell-1\right)}}\right. \\
\left.\left(x-\frac{\omega a_{2}-2 a_{1} \kappa-\alpha}{1-a_{2} \kappa} t\right), \ell\right] \cdot e^{i(-\kappa x+\omega t+\theta)} .
\end{gathered}
$$

In Eq. (24), when the modulus $\ell \rightarrow 1$, we obtain following dark optical soliton solution

$$
\begin{gathered}
q(x, t)=\sqrt{\frac{2\left(\omega+a_{1} \kappa^{2}-\omega a_{2} \kappa+\alpha \kappa\right)}{\kappa \sigma+b-\kappa \lambda}} \sec h\left[\sqrt{\frac{\omega+a_{1} \kappa^{2}-\omega a_{2} \kappa+\alpha \kappa}{a_{1}-a_{2} v}}\right. \\
\left.\left(x-\frac{\omega a_{2}-2 a_{1} \kappa-\alpha}{1-a_{2} \kappa} t\right)\right] \cdot e^{i(-\kappa x+\omega t+\theta)} .
\end{gathered}
$$

with conditions

$$
\begin{gathered}
\left(\omega+a_{1} \kappa^{2}-\omega a_{2} \kappa+\alpha \kappa\right)(\kappa \sigma+b-\kappa \lambda)>0 \\
\left(\omega+a_{1} \kappa^{2}-\omega a_{2} \kappa+\alpha \kappa\right)\left(a_{1}-a_{2} v\right)>0
\end{gathered}
$$

Now, if we use the assumption

$$
u(\xi)=A n s^{\rho}(B \xi, \ell)
$$

From (28), Eq. (9) reduces to

$$
\begin{aligned}
& \left(a_{1}-a_{2} v\right)(\rho-1) \rho \ell^{2} A B^{2} n s^{\rho-2}(B \xi, \ell) \\
& -\left(a_{1}-a_{2} v\right) \rho^{2}\left(1+\ell^{2}\right) A B^{2} n s^{\rho}(B \xi, \ell) \\
& +\left(a_{1}-a_{2} v\right) \rho(\rho+1) A B^{2} n s^{\rho+2}(B \xi, \ell) \\
& -\left(\omega+a_{1} \kappa^{2}-\omega a_{2} \kappa+\alpha \kappa\right) A n s^{\rho}(B \xi, \ell) \\
& +(\kappa \sigma+b-\kappa \lambda) A^{3} n s^{3 \rho}(B \xi, \ell)=0 .
\end{aligned}
$$

When the modulus $\ell \rightarrow 1$ in Eq. (29), we obtain following singular optical soliton solution

$$
\begin{gathered}
q(x, t)=\sqrt{\frac{\omega+a_{1} \kappa^{2}-\omega a_{2} \kappa+\alpha \kappa}{\kappa \sigma+b-\kappa \lambda}} \operatorname{coth}\left[\sqrt{\frac{\omega+a_{1} \kappa^{2}-\omega a_{2} \kappa+\alpha \kappa}{2\left(a_{1}-a_{2} v\right)}}\right. \\
\left.\left(x-\frac{\omega a_{2}-2 a_{1} \kappa-\alpha}{1-a_{2} \kappa} t\right)\right] . e^{i(-\kappa x+\omega t+\theta)} .
\end{gathered}
$$

with conditions (26) and (27).

\section{Conclusion}

This paper consider FLE with full nonlinearity and perturbation terms. Jacobi elliptic functions are used for the integration scheme here. Bright and dark and singular optical soliton solutions are obtained using three types Jacobi 
elliptic functions. The existence criteria of these solutions are also indicated.These solutions provide recognise physical phenomena described by the equation.Due to the fact that bright and dark optical soliton solutions always help to address the soliton dynamics in long distance telecommunication system, the results of the paper are useful in the fiber optics communication technology. It can be obtained more solutions of the FLE using the other Jacobi elliptic functions. This technique is very useful and effective to get soliton solutions of nonlinear partial differential equations in mathematical physics.

Fuzzy logic can be a useful tool for the navigation process in the distributed MRS and more particularly in a dynamic and uncertain environment. It can be used to formulate the different information received and observed by robots in the form of linguistic terms similar to those used by human. From this point of view, we can design robots with reasoning abilities which are similar to those found in humans. This may be possible if we choose a set of rules that allow the use these linguistic variables to conclude other information in order to make a particular decision. For this, it is necessary to use an appropriate architecture, especially when the functionalities of the robots are effectively separated. The process of selection behaviours proposed shows a great flexibility to implement the different navigation approaches in multi-robot systems.

\section{Competing interests}

The authors declare that they have no competing interests.

\section{Authors' contributions}

All authors have contributed to all parts of the article. All authors read and approved the final manuscript.

\section{References}

[1] Biswas, A., Yildirim, Y., Yasar, E., Zhou Q., Moshokoa S.P., Belic M. (2018). Optical soliton solutions to Fokas-Lenells equation using some different methods, Optik-Intern. Journ. for Light and Electron Optics, 173, 21-31.

[2] Arshed, S., Raza, N. (2020). Optical solitons perturbations of Fokas-Lenells equation with full nonlinearity and dual dispersion, Chinese Journal of Physics, 63, 314-324.

[3] Ates, E., Inc, M. (2017). Travelling wave solutions of generalized Klein-Gordon equations using Jacobi elliptic functions. Nonlinear Dyn, 88, 2281-2290.

[4] Inc, M., Ates, E., Tchier F. (2016 ). Optical solitons of the coupled nonlinear Schrodinger's equation with spatiotemporal dispersion, Nonlinear Dyn, 84 (2), 1-11.

[5] Kudryashov, N.A., (2019). First integrals and general solution of the Fokas-Lenells equation, Optik-Int. Journ. for Light and Electron Optics, 195, 163135.

[6] Biswas, A., Ekici, M., Sonmezoglu, A., Alqahtani, R.T., (2018). Optical soliton perturbation with full nonlinearity for FokasLenells equation, Optik 165, 29-34.

[7] Triki, H., Wazwaz, A.M., (2017). New types of chirped soliton solutions for the Fokas-Lenells equation, Int. J. Numer. Methods Heat Fluid Flow 27 (7), 1596-1601.

[8] Liu, F., Zhou, C.C., Lü, X., Xu, H., (2020). Dynamic behaviors of optical solitons for Fokas-Lenells equation in optical fiber, Optik $224,165237$.

[9] Sajid N., Akram G., (2021). Dark, singular, bright, rational and periodic solutions of the space-time fractional Fokas-Lenells equation by the $\phi^{6}$-model expansion method, Optik 228165843.

[10] Bulut, H., Slaiman, T.A., Baskonus, H.M., Rezazadeh, H., Eslami, M., Mirzazadeh, M. (2018). Optical solitons and other solutions to the conformable space-time fractional Fokas-Lenells equation, Optik-Intern. Journ. for Light and Electron Optics 172, $20-27$. 
[11] Ali, K.K., Osman, M.S., Abdel-Aty, M. (2020). New optical solitary wave solutions of Fokas-Lenells equation in optical fiber via Sine-Gordon expansion method, Alexandria Engin. Journ. 59 (3), 1191-1196. 